\title{
Noninvasive diagnostic criteria for hepatocellular carcinoma
}

\author{
Han Chu Lee \\ Department of Internal Medicine, Asan Medical Center, University of Ulsan College of Medicine, Seoul, Korea
}

Keywords: Carcinoma, Diagnosis; Guideline; Hepatocellular

\section{See Article on Page 185}

Hepatocellular carcinoma (HCC) is unique in that its diagnosis generally involves the use of noninvasive rather than histological criteria. The rationale underlying the preference for noninvasive diagnostic criteria by hepatologists for defining the presence of HCC include the well-defined risk groups for its development, bleeding diathesis in cirrhotic patients, technical difficulties in certain locations, difficulties in differentiation between malignant and benign lesions even with a biopsy specimen, and the existence of characteristic serum tumor markers and radiological findings. However, noninvasive diagnosis must be implemented with care, since the noninvasive diagnostic criteria inevitably carry a risk of falsepositive HCC diagnosis.

In general, the sensitivity, specificity, positive predictive value (PPV), negative predictive value (NPV), and diagnostic likelihood ratios are used to evaluate the accuracy of a diagnostic test or criterion, depending upon the purpose of that test or criterion and its relative importance. For example, the optimal level of serum alpha-fetoprotein (AFP) during a surveillance program is determined by the areas under the sensitivity and 1-specificity curves. In other words, to determine whether a test is useful as a surveil- lance test, the most important factors are sensitivity with an acceptable specificity trade-off. However, if serum AFP level is used for making a definite diagnosis of HCC in a patient with a hepatic mass or nodule, the concept should be changed. Since most treatments for cancer carry substantial treatment-related morbidities and mortalities and the treatment strategy may differ depending on the type of cancer, theoretically there should be no false-positive cases. Therefore, the PPV and specificity should be very close to $100 \%$. In other words, a biopsy can be skipped only when the possibility of HCC is nearly $100 \%$. In this case, the NPV is less important, since these patients should be biopsied anyway in order to diagnose a hepatic mass. For example, an NPV of 100\% means that the patient does not have HCC if the test result is negative; however, there will still be a chance of malignant disease other than HCC. Therefore, we can say that a test or criterion is superior to others only if the sensitivity can be increased while maintaining the PPV or specificity at $100 \%$.

Bias may occur if a test or criterion is evaluated under conditions that do not reflect the real clinical situation for which it is being used. In the study of Bae et al., about $80 \%$ of the studied patients underwent surgical resection or liver transplantation; patients who had multiple tumors were excluded. Therefore, most

\footnotetext{
Abbreviations:

AASLD, American Association for the Study of Liver Diseases; AFP, alpha-fetoprotein; CEUS, contrast-enhanced ultrasonography; $\mathrm{CT}$, computed tomography; FNH, focal nodular hyperplasia; HCC, hepatocellular carcinoma; MRI, magnetic resonance imaging; NCCN, National Comprehensive Cancer Network; NPV, negative predictive value; PPV, positive predictive value; USG, ultrasonography
}

\section{Corresponding author : Han Chu Lee \\ Department of Internal Medicine, Asan Medical Center, University of Ulsan College of Medicine, 88 Olympic-ro 43-gil, Songpa-gu, Seoul 138-736, Korea \\ Tel. +82-2-3010-3190, Fax. +82-2-485-5782, E-mail; hch@amc.seoul.kr}


of the studied patients had a single tumor or tumors at an early stage. In addition, only 72 patients underwent a liver biopsy. Given that this study was performed at a large-volume center for HCC, these patients might represent less than $10 \%$ of all patients who attended the center during the 2-year study period. Therefore, the basic question is whether or not these studied patients represent all patients with hepatic nodule(s) or mass(es) in a hepatology department.

Several benign or malignant tumors can mimic the typical enhancement pattern (arterial hypervascularity and portal/delayed washout) of HCC, leading to the following possible differential diagnoses: focal nodular hyperplasia (FNH), or FNH-like nodules, ${ }^{2}$ multiacinar regenerative nodule (macroregenerative nodule), ${ }^{3}$ angiomyolipoma of the liver, ${ }^{4}$ hepatocholangiocarcinoma, atypical cholangiocarcinoma (on contrast-enhanced ultrasound, CEUS), and benign or malignant tumors of neuroendocrine origin. ${ }^{6}$ Furthermore, elevated AFP levels can sometimes be observed in cholangiocarcinoma, hepatocholangiocarcinoma, metastatic adenocarcinoma of gastrointestinal origin including the stomach, colon, or pancreas, or germ-cell tumor. ${ }^{6,7}$ In addition, incidental benign nodule(s) or tumor(s) may be found in patients with active necroinflammation and elevated AFP levels.

Thus, we can assume that some patients might not have received a liver biopsy and would thereby have been excluded from the study of Bae et al.' (considering the relatively low rate of biopsied cases in that study), such as patients at a high risk of HCC with a typical enhancement pattern or with elevated serum AFP levels. Therefore, false-positive cases yielded from dynamic computed tomography (CT) or elevated serum AFP levels in highrisk patients would be underestimated with their study design, since most of those patients may not have received a liver biopsy. To avoid this selection bias, consecutive patients should be enrolled for evaluating the noninvasive diagnostic criteria for HCC. In other words, to evaluate the diagnostic accuracy of a test(s), all of the studied patients should receive the index test (here, dynamic CT or serum AFP) as well as a test(s) for a reference standard (pathological diagnosis). In the study of Bae et al.," nearly all of the patients received the index tests, but only a small proportion received the test for the reference standard, which is prone to many types of bias. There remains a possibility of selection bias even when surgical resection is performed without a preoperative fine-needle biopsy, as for most of the cases in that study. This is because most false-positive cases yielded by dynamic imaging studies or elevated serum AFP levels have metastatic tumors, cholangiocarcinoma, or hepatocholangiocarcinoma, as discussed above, which are usually inoperable at the time of diagnosis. ${ }^{6}$ Furthermore, FNH-like nodules or regenerative nodules in alcoholic cirrhosis or hepatic outflow obstruction are usually multiple. ${ }^{2}$ In contrast, patients who undergo surgical resection usually have a single tumor or have early-stage disease. In addition, the absence of dysplastic nodules in that series suggests that a liver biopsy, surgery, or liver transplantation was performed in highly suspected cases of $\mathrm{HCC}$ when the nodule diameter was between 1 and $2 \mathrm{~cm}$. Therefore, the study of Bae et al.' excluded not only patients with advanced disease but also those with very early disease, which could adversely inflate the diagnostic accuracy of the criteria being tested (limited challenge bias). It is therefore very difficult to draw a conclusion regarding the best diagnostic criteria from that study. Another weak point of that study is that what cases resulted in false-positive HCC diagnoses with each diagnostic criterion was not reported.

Bae et al.' evaluated the diagnostic accuracy of past guidelines from the American Association for the Study of Liver Diseases (AASLD) and the National Comprehensive Cancer Network (NCCN), which have since been updated. The salient points of these guidelines vary. First, the importance of portal/delayed washout is emphasized in newer guidelines. The European Association for the Study of the Liver guideline is no longer accepted in this regard. Second, serum AFP is no longer used as a diagnostic criterion, ${ }^{8}$ since there can be several false-positive cases, as discussed above. Interestingly, there is no cut-off level for serum AFP that could be used to completely avoid errors in diagnosis. ${ }^{6}$ Another important problem is that most false-positive cases are cholangiocarcinoma or hepatocholangiocarcinoma, which share risk factors for HCC in their development, ${ }^{9}$ thus raising questions regarding the use of serum AFP as a diagnostic criterion even in cirrhotic patients. However, this problem is minimized in the Korean Liver Cancer Study Group/National Cancer Center guidelines, ${ }^{10}$ since the diagnosis of HCC requires both a typical enhancement pattern of dynamic imaging studies and elevated serum AFP level. Third, the recent 2010 AASLD guidelines no longer adopted CEUS as a standard dynamic imaging study, since the PPV is relatively low $(75 \%)^{11}$ and cholangiocarcinoma can mimic the typical CEUS enhancement pattern. ${ }^{5}$ The 2005 AASLD guidelines required a typical enhancement pattern by two dynamic imaging studies for the diagnosis of HCC between 1 and $2 \mathrm{~cm}$ in diameter. However, the 2010 AASLD guidelines suggested that HCC can be diagnosed if there is a typical enhancement pattern in one dynamic imaging study, even in nodules between 1 and $2 \mathrm{~cm}$ that are detected on surveillance ultrasonography (USG) in cirrhotic patients. In fact, the validation 
study for the 2005 AASLD guidelines considered only a single false-positive case (regenerative nodule) identified by dynamic magnetic resonance imaging (MRI). ${ }^{3}$

When two scan types (dynamic MRI and CEUS) were combined and both were required to be positive, the PPV and specificity were $100 \%$, but the sensitivity dropped from $61.7 \%$ with dynamic MRI alone to $33.3 \%$ with the MRI/CEUS combination. ${ }^{3}$ Another recent study also showed that the typical enhancement pattern on dynamic MRI scan is highly specific, with a $100 \%$ specificity and PPV. Dynamic CT yielded a 95\% PPV and 99\% specificity. Sequential imaging can increase the sensitivity up to $74 \%$, but with slightly diminished specificity (99\%) compared to MRI alone." Therefore, the 2010 AASLD guidelines recommend the sequential approach, combined with a liver biopsy if the dynamic imaging scan(s) does(do) not reveal a typical enhancement pattern. However, this approach can produce false-positive cases (although they are rare, at $<5 \%$ ), since the PPV of dynamic MRI or CT scan is not $100 \%$.

Fourth, there is still no clear recommendation as to whether these diagnostic criteria are only applicable to nodules detected on surveillance USG in cirrhotic patients or can be expanded to mass(es) or nodule(s) detected on the first surveillance USG or incidentally in (noncirrhotic) high-risk patients. The recent NCCN guidelines $^{12}$ suggested that these diagnostic criteria are applicable to incidental nodule(s) or mass(es) in noncirrhotic, high-risk patients, but the only supporting data come from a single study involving a mass of $>2 \mathrm{~cm} .{ }^{6}$ Since the PPV of a test is influenced by the disease prevalence, the incidence of HCC should be sufficiently high to minimize the number of false-positive cases. In addition, a new lesion that develops during USG-based surveillance suggests that there is a low probability of benign nodules such as FNH, angiomyolipoma, and adrenal rest tumor. Therefore, the diagnostic accuracy of a test can differ between incidental masses found in noncirrhotic, high-risk patients and masses found in cirrhotic patients on surveillance USG. Care must therefore be taken when evaluating an incidental mass detected in a noncirrhotic, high-risk patient.

Theoretically, about $5-7 \%$ of Korean patients with benign nodules mimicking $\mathrm{HCC}$ can be positive for HBsAg. For example, the risk of a 35-year-old female in an HBeAg-negative healthy carrier state developing HCC would be similar to that for the general population. ${ }^{13,14}$ An incidental nodule or mass found in such a patient would have a relatively high probability of benign or malignant disease other than HCC, and therefore should be biopsied. However, the level of incidence at which the PPV becomes acceptable in noncirrhotic, high-risk patients is still unknown.

Finally, although there is no mention in the guidelines, nodules or masses found in hepatic outflow obstruction such as Budd-Chiari syndrome, membranous obstruction of the inferior vena cava, or constrictive pericarditis should be biopsied regardless of the enhancement pattern, since the incidence of benign regenerative nodules is quite high and the enhancement patterns sometimes cannot be distinguished from those of HCC., 2,15

In conclusion, although there have been many guidelines for noninvasive $\mathrm{HCC}$ diagnostic criteria, a recent trend is that the recognition of a typical enhancement pattern on one dynamic imaging study (especially dynamic MRI) is sufficient for diagnosing $\mathrm{HCC}$ if a nodule larger than $1 \mathrm{~cm}$ is detected on the surveillance tests in cirrhotic patients. A combination of two dynamic imaging techniques can increase the PPV and specificity, but the sensitivity drops dramatically, with more patients requiring a biopsy. Furthermore, combining two dynamic imaging techniques can increase the medical cost. There is still no clear answer as to whether this updated guideline is applicable to incidental nodules of $1-2 \mathrm{~cm}$ detected in noncirrhotic patients at a high risk of HCC. Therefore, a well-designed study is mandatory to draw a conclusion regarding the best diagnostic criteria.

\section{Conflicts of Interest}

The authors have no conflicts to disclose.

\section{REFERENCES}

1. Bae SY, Choi MS, Gwak GY, Paik YH, Lee JH, Koh KC, et al. Comparison of usefulness of clinical diagnostic criteria for hepatocellular carcinoma in a hepatitis B endemic area. Clin Mol Hepatol 2012;18:185-194.

2. Choi JY, Lee HC, Yim JH, Shim JH, Lim YS, Shin YM, et al. Focal nodular hyperplasia or focal nodular hyperplasia-like lesions of the liver: a special emphasis on diagnosis. J Gastroenterol Hepatol 2011;26:1004-1009.

3. Forner A, Vilana R, Ayuso C, Bianchi L, Solé M, Ayuso JR, et al. Diagnosis of hepatic nodules $20 \mathrm{~mm}$ or smaller in cirrhosis: Prospective validation of the noninvasive diagnostic criteria for hepatocellular carcinoma. Hepatology 2008;47:97-104.

4. Yang B, Chen WH, Li QY, Xiang JJ, Xu RJ. Hepatic angiomyolipoma: dynamic computed tomography features and clinical correlation. World J Gastroenterol 2009;15:3417-3420.

5. Vilana R, Forner A, Bianchi L, García-Criado A, Rimola J, de Lope $C R$, et al. Intrahepatic peripheral cholangiocarcinoma in cirrhosis 
patients may display a vascular pattern similar to hepatocellular carcinoma on contrast-enhanced ultrasound. Hepatology 2010;51:2020-2029.

6. Kim SE, Lee HC, Shim JH, Park HJ, Kim KM, Kim PN, et al. Noninvasive diagnostic criteria for hepatocellular carcinoma in hepatic masses $>2 \mathrm{~cm}$ in a hepatitis B virus-endemic area. Liver Int 2011;31:1468-1476.

7. Isonishi S, Ogura A, Kiyokawa T, Suzuki M, Kunito S, Hirama M, et al. Alpha-fetoprotein (AFP)-producing ovarian tumor in an elderly woman. Int J Clin Oncol 2009;14:70-73.

8. Bruix J, Sherman M; American Association for the Study of Liver Diseases. Management of hepatocellular carcinoma: an update. Hepatology 2011;53:1020-1022.

9. McGlynn KA, Tarone RE, El-Serag HB. A comparison of trends in the incidence of hepatocellular carcinoma and intrahepatic cholangiocarcinoma in the United States. Cancer Epidemiol Biomarkers Prev 2006;15:1198-1203.

10. Park JW, Choi JY, Seo KS. 2009 Hepatocellular carcinoma practice guideline. Seoul: The Korean Liver Cancer Study Group and the Na- tional Cancer Center, 2009:1-56.

11. Khalili K, Kim TK, Jang HJ, Haider MA, Khan L, Guindi M, et al. Optimization of imaging diagnosis of $1-2 \mathrm{~cm}$ hepatocellular carcinoma: an analysis of diagnostic performance and resource utilization. J Hepatol 2011;54:723-728.

12. Benson AB 3rd, Abrams TA, Ben-Josef E, Bloomston PM, Botha $J F$, Clary BM, et al. NCCN clinical practice guidelines in oncology: hepatobiliary cancers. J Natl Compr Canc Netw 2009;7:350-391.

13. Yang HI, Sherman M, Su J, Chen PJ, Liaw YF, Iloeje UH, et al. Nomograms for risk of hepatocellular carcinoma in patients with chronic hepatitis B virus infection. J Clin Oncol 2010;28:2437-2444.

14. Yang HI, Lu SN, Liaw YF, You SL, Sun CA, Wang LY, et al. Hepatitis $B$ e antigen and the risk of hepatocellular carcinoma. N Engl J Med 2002;347:168-174

15. Gwon D 2nd, Ko GY, Yoon HK, Sung KB, Kim JH, Lee SS, et al. Hepatocellular carcinoma associated with membranous obstruction of the inferior vena cava: incidence, characteristics, and risk factors and clinical efficacy of TACE. Radiology 2010;254:617-626. 